\title{
PERÓN EN EL CONGRESSO: UM ANÁLISIS DISCURSIVO DE LAS APERTURAS DE SESSIONES ORDINARIAS DURANTE EL PERONISMO HISTÓRICO(1946-1955)
}


PERÓN EN EL CONGRESSO: UM ANÁLISIS DISCURSIVO DE LAS APERTURAS DE SESSIONES ORDINARIAS DURANTE EL PERONISMO HISTÓRICO(1946-1955)

Resúmen:

En el presente trabajo se estudiarán las representaciones sobre la Nación Argentina y la tensión con las regiones del país. Se realizará a través de los mensajes con los que el presidente Juan Domingo Perón inaugura las sesiones ordinarias del Congreso nacional en tiempos del llamado peronismo histórico entre 1946 y 1955. El objetivo del trabajo es mostrar cómo el discurso peronista pretende poner fin a un país con históricas desigualdades territoriales, promoviendo, en cambio, un proceso de integración, equilibrio y homogenización regional.

Palabras Clave: Peronismo; Nación; Discurso; Congreso

\title{
PERÓN NO CONGRESSO: UMA ANÁLISE DISCURSIVA DAS ABERTURAS DE SESSÕES ORDINÁRIAS DURANTE O PERONISMO HISTÓRICO(1946-1955)
}

\section{Resumo:}

No presente trabalho estudar-se-ão as representações sobre a Nação Argentina e a tensão com as regiões do país. Realizar-se-á através das mensagens que o presidente Juan Domingo Perón inaugura as sessões ordinárias do Congresso nacional em tempos do chamado peronismo histórico entre 1946 e 1955 . O objetivo do trabalho é mostrar como o discurso peronista pretende pôr fim a um país com históricas desigualdades territoriais, promovendo, em mudança, um processo de integração, equilíbrio e homogenização regional.

Palavras chave: Peronismo; Nação; Discurso; Congresso

\section{PERÓN IN CONGRESS: A DISCURSIVE ANALYSIS OF THE OPENING OF ORDINARY SESSIONS DURING THE HISTORICAL PERONISM (1946-1955)}

\begin{abstract}
:
In the present work will be studied the representations about the Argentine Nation and the tension with the regions of the country. It will perform through the messages that the president Juan Sunday Perón opens the ordinary sessions of the national Congress in time of the called historical peronism between 1946 and 1955. The aim of the work is to show how the peronist speech intends to put end a country with historical territorial inequalities, promoting, in changing, a process of integration, balance and regional homogenization.
\end{abstract}

Keywords: Peronismo; Nation; Speech; Congress 


\section{MARCO TEÓRICO Y CONTEXTO HISTÓRICO}

El discurso peronista del periodo 1946 - 1955 presenta una particular construcción discursiva que sostiene el proceso de integración nacional y homogenización regional. La "Nueva Argentina" pareciera orientarse hacia la realización de un programa capaz de equilibrar las diferentes realidades regionales.

El objetivo de este trabajo, por tanto, es reflexionar sobre los discursos de los primeros gobiernos populistas peronistas e indagar si efectivamente subordinan al Poder Ejecutivo la acción deliberativa, que se realiza en el marco del Poder Legislativo. Se analizarán los mensajes de apertura de las sesiones ordinarias del Congreso de la Nación Argentina, en tiempos del peronismo histórico.

Se prestará especial atención a aquello que Perón dice y lo que silencia vinculado a un tema medular para un país de casi 3 millones de kilómetros cuadrados con desigualdades territoriales: la tensión entre región y Nación. Para lograr este fin, se pondrá especial atención al desarrollo del marco teórico. Se partirá de un análisis discursivo comunicacional mediante el cual el lenguaje es entendido como parte de un dispositivo ideológico, que estructura las relaciones sociales y que es puesto en interacción por los sujetos en una sociedad, en el que se tendrá en cuenta el tema de poder y con él un modelo particular de discurso -el discurso político-. Este entramado genera en la sociedad argentina un tipo de movimiento populista con características muy específicas y a su vez, se desenvuelve de un modo especial dadas las lógicas regionales del país. Es importante destacar que este Estado nacional, popular, presidencialista y vertical expone una determinada relación con la máxima instancia legislativa.

El lenguaje ${ }^{1}$ facilitaría la comunicación y posibilitaría el armado y el intercambio de representaciones sociales entre los sujetos de una comunidad. Las representaciones sociales se realizarían en y mediante el discurso². Estos últimos vehiculizarían las interpretaciones necesarias para la vida en sociedad; además las prácticas sociales son las que permiten la circulación de ideologías y jerarquizan los acontecimientos ${ }^{3}$.

1El lenguaje funciona como una herramienta cognitiva que permite la formación y la complejización de las representaciones y posibilita la transmisión y el intercambio de ellas entre los hombres. "Es, por medio del lenguaje que las representaciones no están limitadas a ser de algún modo un reflejo del mundo que los rodea" porque los seres humanos mediante las representaciones dan sentido, "completan" el mundo. (Raiter, Alejandro (2002), pag 12-13) 2 "[...] el discurso -entendido como todo producto simbólico- no sólo es una expresión de las representaciones sociales sino que es constitutivo de las representaciones sociales" (Neyra Pardo Abril (2003), pag 62)

3Alejandro Raiter subraya que las representaciones dan cohesión si son socialmente compartidas en la comunidad. Algunas se transforman en leyes. (Raiter, Alejandro (2002)). Neyra Pardo Abril, por su parte destaca que las representaciones sociales se concretan mediante el discurso, porque sus elementos constituyentes son "lo suficientemente diversos para lograr 'realidad social'” (Neyra Pardo Abril (2003)) 
Un tipo particular de discurso es el político 4 que se relaciona con la noción de populismo, dado que en este tipo de vínculo, el líder -un emisor-pretende persuadir a los destinatarios -múltiples y que evaluarían los dichos del emisor-.

El populismo ${ }^{5}$ se centraría en un liderazgo respecto de la figura de un pueblo como conjunto de actores, como conglomerado ${ }^{6}$. El líder, desde el discurso, manejaría la exclusión del enemigo o del adversario, así como en este espacio discursivo mostraría y silenciaría el origen propio, de acuerdo al contexto y al impacto que desee causar. Una característica distintiva sería la comunicación colectiva7 entre el líder y el pueblo, más aun si hay una elección del líder emisor de utilizar la primera persona en el discurso, ya que se convertiría en el foco, en lugar de ser el representante del pueblo.

El líder llegaría al poder, al gobierno para redimir al pueblo ${ }^{8}$, para salvarlo de los enemigos que lo acosan. Su llegada aparece, por lo tanto, como un sacrificio y no como una obligación cívica?.

En la construcción del imaginario populista habría puntos claves sobre los que se montan las lógicas y los discursos del líder, a veces están vaciados de contenido mientras que en otros casos son polivalentes ${ }^{10}$. Una manera interesante de entender esta cuestión es mediante la propuesta de análisis atractiva de Verón y Sigal'1". Estos autores estudian el fenómeno peronista desde la enunciación y focalizan en la figura de un enunciador abstracto ${ }^{12}$ que puede cambiar su ubicación de acuerdo al modo de presentación y propia construcción en el discurso.

Es importante señalar que en el caso del populismo, la representación

4 "[... el discurso político es la clase de textos, con la intencionalidad (manifiesta o encubierta) de producir un cambio social, realiza una labor de persuasión hacia los destinatarios que construye, con el objetivo de producir cambios. Su éxito depende de múltiples actores, pero no hacen a la definición de discurso político" Este discurso se puede definir como el medio por el cual se realizan intercambios de imaginarios ideológicos, se determinan los valores de los signos ideológicos (intercambiándose esos valores, e imponiéndose otros). El discurso político para Alejandro Raiter provoca un cambio, genera una conducta y una creencia social. Es el emisor de discursos que persigue y obtiene esos cambios, y quien expresa sus creencias. A su vez, frente a sí aparecen una variedad de receptores -pluralidad de receptores evaluadores-, que pueden ser partidarios, adversarios o indiferentes a esos discursos. Estos son los que posibilitan cambio de conductas o creencias. (Raiter, Alejandro(1994))

5Confrontar: Aboy Carlés, Gerardo. (2005); Madriz, María Fernanda (2002); Verón, E. - Sigal, S. (2003); Lattuada, Mario (2002); De Ipola, (1980), (1983); Álvarez Junco y González Leandri, (1994)

6Aboy Carlés, Gerardo (2005)

7 Madriz, María Fernanda (2002)

8 Verón, E. - Sigal, S. (2003)

9 Madriz, María Fernanda. (2002)

10 Madriz, María Fernanda. (2002)

11 Verón, E. - Sigal, S. (2003)

12 Verón, E. - Sigal, S. (2003) 
del Otro aparecería como la del adversario, del enemigo ${ }^{13}$. Algunos autores los denominan como fuerzas ocultas u obscuras -sombras ${ }^{-14}$, con una posición desplazada, ya que en los populismos y en el peronismo, los enemigos y el líder no están, no aparecen en el mismo plano, ya que sólo el líder tiene voz.

Ernesto Laclau mostró como característica definitoria del populismo la dimensión rupturista. Por su parte, Emilio de Ipola y Juan Carlos Portantiero afirmaron que el populismo existe como proyecto y "acaba por cerrar y coartar su propia conflictividad inicial derivando en la integración de un nuevo orden de tipo organicista que desactiva el potencial de ruptura"15. También es importante recordar, el aporte de Mario Lattuada mediante el que especifica que el fenómeno populista ha sido observado desde una perspectiva sincrónica y desde una perspectiva histórica, diacrónica así el autor hace distinciones en un campo político, un campo social, un campo económico, un campo ideológico ${ }^{16}$.

Es importante destacar que habitualmente se caracteriza al peronismo histórico (1946-1955), como una variante de populismo urbano, promotor de una política económica planificada, dirigista y nacionalista, con apoyo y base en una fuerte alianza con el movimiento obrero y la pequeña y mediana burguesía nacional ${ }^{17}$.

Una particularidad a la que se hacía referencia respecto del peronismo era el uso de un lenguaje político tradicional, reconocido por el 'pueblo'. Si se recurre al análisis desde la enunciación pueden observarse dos entidades: enunciador, en este caso el líder, la construcción que Perón hace de si mismo en el discurso y el destinatario, el pueblo, aunque también debería incluirse a los `enemigos'18.

Tal como se había comentado, las ideologías, que se expresarían mediante el discurso, y serían, interpretando a T. Van Dijk ${ }^{19}$, sistemas de creencias, valores, representaciones que influirían y contribuirían a dar legitimidad y reproducir normas, principios así como las relaciones sociales en una sociedad. A su vez, las ideologías servirían para mantener, crear y transformar las relaciones de poder y control ${ }^{20}$.

13Verón, E. - Sigal, S. (2003)

14 "[... ] la estrategia retórica que permite al discurso populista persuadir al pueblo de que se encuentra amenazado, es la construcción discursiva de lo que he definido como "las fuerzas"”. Estas son artificios retóricos del líder para amedrentar a ‘el soberano [pueblo]’. Estas entidades están ocultas, no portan rostro ni nombre, pero tienen, según la autora "un alto potencial de destrucción que, agazapadas, mantienen bajo zozobra al pueblo y sus dirigentes. [... ] Así, las fuerzas brindan al imaginario populista magros servicios. [... son] como una suerte de caparazón vacío que no refiere a ninguna persona o grupo en concreto [... ] permiten al líder populista proponerse como imbatible redentor del soberano [pueblo], como paladín de los desvalidos, como infalible conjuro ante todas las conjuras" (Madriz, María Fernanda (2002)) 15En Aboy Carlés, Gerardo. (2005), pag 4.

16 Lattuada, Mario (2002)

17 De Ipola, (1980), (1983); Álvarez Junco y González Leandri, (1994)

18 Verón, E. - Sigal, S. (2003) y Madriz, María Fernanda (2002)

19Van Dijk, Teun A. (2004) y Van Dijk, Teun. (2005)

20Pardo Abril, Neyra (2003), pag 58 
Es importante destacar que el poder, es un concepto central ya que se halla disperso en todas las capas de la sociedad y se trasporta y ejerce mediante el discurso ${ }^{21}$. Por lo tanto, en virtud del ejercicio del poder, pueden observarse en el discurso procedimientos de inclusión y de exclusión para controlar los poderes desestabilizadores. Los binomios "normal-habitual” y "anormal-inhabitual" se vincularían con las nociones de "nosotros" y "ellos", así se intentan exponer nuestras cosas buenas y sus aspectos malos (los de ellos, de los Otros)22.

Mediante las ideologías compartidas y la construcción del nosotros se definiría la identidad de un grupo y la noción de pertenencia, ya que se mostrarían los valores culturales que son importantes para el grupo ${ }^{23}$.

El peronismo logró instalarse dado que su retórica sobre la justicia social y la soberanía nacional eran temas verosímilmente interrelacionados antes que consignas abstractas meramente enunciadas y ya conocidas con anterioridad, es decir, instaladas en la sociedad previamente ${ }^{24}$.

Es importante remarcar que la Nación se constituiría como tal en tanto presenta un territorio delimitado, sobre él hay habitantes y están reglados por leyes que son administradas por un Estado. En el caso que se presenta, se trata de un Estado federal, que genera y es parte de las relaciones sociales. Ahora bien, desde la cuestión económica, la Argentina está atravesada por lógicas capitalistas, que forman parte de un sistema que tiene capacidades para apropiarse no sólo del espacio sino de aquello que la sociedad genera.

El espacio sería una construcción social generada por grupos sociales a lo largo del tiempo y el territorio si bien se constituiría por elecciones sociales, lo sería marcadamente por decisiones políticas. Así las regiones resultarían de procesos territoriales y responderían a ideologías, estarían surcadas por la idea de poder. De manera que las regiones serían la resultante de interrelaciones económicas y sociales ${ }^{25}$.

Para ingresar en el estudio, según Juan Domingo Perón en 1945: "No lleguemos a pensar que el Estado es todo y los individuos nada, porque el todo es la Nación, y el Estado es, dentro de ella, una sola de sus partes" ${ }^{26}$. Mediante esta reflexión se puede observar que se hace hincapié en la construcción de Estado como parte de la idea de Nación y en conjunto con los individuos, las leyes, el territorio. El territorio, por cuestiones socio-político-económicas, se habría dividido en regiones, ahora bien, el peronismo parece presentar en sus construcciones discursivas un acercamiento a la noción de nación como unidad y no como conjunto de regiones distintas.

Vale destacar que "[... ] la expansión de derechos sociales del peronismo

21Foucault (1999) en Pardo Abril, Neyra (2003), pag 58

22 Martín Rojo, Luisa (1997), pag 16, 17, 18

23 Van Dijk, Teun A. (2004), pag 27, 28

24 James, Daniel (2006) pag 35

25 Girbal-Blacha, Noemí. (1997), pág 227

26Perón, Juan D. 18/enero/1945. Límites del Estado. Pág. 34 
estuvo marcada por la impronta de una homogeneidad territorial desconocida que llegó a constituir una sociedad más plenamente integrada y menos diferenciada en términos de disfrute de estos derechos, desde La Quiaca hasta Tierra del Fuego."27

El movimiento peronista se conformó con dirigentes de sindicatos, militantes del sector obrero, nuevos trabajadores, migrantes y sectores de pocos recursos, así como vieja clase obrera y nuevos trabajadores industriales. Su construcción partió desde la denuncia discursiva al sistema democrático formal, aunque se haya abrevado de él. Perón mediante el discurso unifica y organiza su 'grupo' y así los dirigentes del primer gobierno trabajaron para la consolidación y expansión del Estado intervencionista. Es importante destacar que las Cámaras contaban con mayoría peronistas pero eran hombres de poca experiencia legislativa. Estos actores en el primer gobierno habrían provocado cambios no sólo en el modo de exposición en estos espacios de poder sino también en la orientación y modo del movimiento en general.

De modo tal que aunque se podría considerar al peronismo como un movimiento populista, las intervenciones del 'pueblo' fueron decisivas y no pasivas. En el segundo gobierno funciona como amalgama la voz y discursos de Perón organizando y puliendo la estructura. En esta segunda instancia los intentos de adoctrinamiento y de homogeneización aparecen más marcados.

El peronismo clásico intenta pues desde el discurso mostrar a la Nueva Argentina como un todo pero mantienen latente una distinción entre dos tipos de espacios. Por un lado, la región pampeana con avances y tecnología, con los cuadros burocráticos, epicentro de las decisiones nacionales. Por otro, el "interior", con economías regionales basadas en agriculturas mono-productoras, más dependientes y atadas a un limitado mercado interno con el agravante de no poder exportar y a su vez, con fuertes diferencias entre sí (Tucumán, Cuyo, Patagonia -aislada y marginal-, Gran Chaco, Chaco Austral).

\section{ANÁLISIS DE LAS PALABRAS PRESIDENCIALES EN LAS APERTURAS DE SESSIONES ORDINARIAS EN EL CONGRESSO}

Las aperturas de sesiones legislativas por el Presidente Perón se podrían agrupar en dos conjuntos: de 1946 hasta $1950^{28}$; y de 1951 a 1955. Esta división se corresponde con los temas o las orientaciones que el Presidente expone en sus discursos. Las nociones de federalismo práctico -idea novedosa y de avanzada- que propone el peronismo y la unidad (y equilibrio) de regiones con 27 Aboy Carlés, Gerardo (2005). Pag 10

28Por un lado, "1950, fue el momento en que la organización partidaria desemboca en la división en ramas: la Rama Sindical, la Rama Política y la Rama Femenina [... a principios de 1950] culmina la primera etapa de la organización del partido, su fase constitutiva". (Mackinnon, Moira. (2002), pag 17 y 22). Por otro, el discurso frente a las Cámaras de 1950 efectúa una evaluación completa del año anterior, 1949, de grandes cambios. 
marcadas diferencias, se enlaza a la reforma constitucional (1949), al Primer Plan Quinquenal, a la 'Vuelta al Campo'29 y a la advocación del año del Gral. José de San Martín (1950).

El año 1950 es un año de quiebre, es allí donde se observa el corte. Mientras en la segunda parte se afianza el concepto de Nación y se unifica el partido y el gobierno como doctrina y propuesta para la acción para otras naciones, el Segundo Plan Quinquenal, el plan económico de 1952, la muerte de Eva Perón y una mayor tensión con grupos de poder.

El presidente Perón, en la etapa 1946-1950, se basa en datos estadísti$\cos ^{30}$ para expresar el desarrollo del país. Le da relevancia a algunas regiones y provincias al tiempo que marca diferencias para recuperar un equilibrio que parece haberse perdido. Prioriza la concreción de obras de aprovechamiento hidráulico dada "la propulsión integral del desenvolvimiento económico de cada región del país, [y] con el fin de evitar la improductividad a que se han visto condenadas costosas obras públicas por haberse omitido adoptar, con visión de conjunto y previsión adecuada, las medidas necesarias para el empleo más racional del agua y de la energía habilitadas por dichas obras" ${ }^{31}$. Es importante destacar que a su vez, durante el periodo de sesiones ordinarias, los diputados exponían con una simpleza sus lugares de pertenencia y solicitaban mejoras concretas para esos espacios ${ }^{32}$.

29 "[... ] desde mediados de los años `40 es el accionar del Estado benefactor, popular, dirigista, nacionalista y planificador, el que -a pesar del intento por diseñar una Argentina acogedora de la pequeña y mediana empresa industrial- termina por alentar desde 1950 `la Vuelta al Campo' y apoya, una vez más, con el crédito oficial a las agroindustrias, las actividades rurales y el cooperativismo agrario. (Girbal-Blacha, Noemí. (2004) pag 175 / 176)

30 "La producción de cereales, oleaginosas, frutas y hortalizas llegó en 1945 a más de 15 millones de toneladas, correspondiendo 9 millones al consumo interno. En cultivos especiales: algodón, caña de azúcar, tabaco, yerba mate y uva, se cosecharon más de 8 millones de toneladas, casi todo destinado al consumo interno. En carnes la producción alcanza a 3602000 toneladas en peso vivo, correspondiendo al consumo interno la mayor parte, $69 \%$ y a la exportación el 31\%”. Discurso presidencial ante Honorables Cámaras de Diputados y Senadores. Perón, 26 de junio de 1946

31 Discurso presidencial ante Honorables Cámaras de Diputados y Senadores. Perón, 26 de junio de 1946. Del mismo modo hace años más tarde: “Los servicios de aguas y desagües fueron atendidos normalmente en todo el territorio de la República, activándose las obras para dotar con estos servicios a nuevos centros poblados, así como para ampliar las instalaciones existentes" (Discurso presidencial ante Honorables Cámaras de Diputados y Senadores. Perón, 1 de mayo de 1949).

"En obras sanitarias, los trabajos de provisión de agua y cloacas beneficiaron en su gran mayoría a las poblaciones del interior del país" (Discurso presidencial ante Honorables Cámaras de Diputados y Senadores. Perón, 1 de mayo de 1952)

32Para dar sólo un ejemplo, el Diputado Jabel Arévalo Cabeza presenta un proyecto de ley para la inversión de \$ 30 mil m/n para instalar oficinas telegráficas en Santa Rosa, departamento de 25 de mayo y en Los Berros, departamento de Sarmiento, ambos en San Juan. El diputado expone en general que "el aislamiento es fatal para las poblaciones", explica las 
Respecto de la tierra, el líder frente a las Cámaras comunica que se "están preparando los medios para facilitar la propiedad de las tierras para aquellos que las trabajan venciendo los peligros que acechan al pequeño propietario o al que desea serlo" 33 . Se les otorgaría entonces a los pequeños propietarios créditos oficiales, se estabilizaría el control de precios y de terrenos arables, "para procurar precios justos al agricultor, tanto para adquirir en propiedad como para arrendar a bajo precio, cuyo plan se completa con la concesión de préstamos a bajo interés" 34 . Se apela a la generalidad, el discurso de Juan D. Perón no da precisiones sobre qué espacios del país y de qué manera se otorgarán. A su vez, permite borrar fronteras y comenzar a imaginar a la Nación como un todo.

Es destacable que en 1947, la propuesta antedicha parece, por un lado haber avanzado y por otro, el orador sólo mantiene la ilusión del colono. Ya que: "La esperanza del colono de ser dueño de la tierra que trabaja se va troncando hoy en una palpable realidad. Merced a la simplificación que se ha logrado en los trámites burocráticos y al ritmo acelerado de trabajo impuesto al personal administrativo y técnico, con lo que se ha conseguido que el ansiado título de propiedad. [Este] arraiga y da bienestar a la familia campesina.

[... Se otorga] ahora -tan pronto el poblador cumpla con sus obligaciones legales- en el mismo terreno junto al 'rancho criollo' [... ] Ya no habrá más 'intrusos' que figuren al margen de la ley, porque se van a convertir paulatinamente en propietarios o arrendatarios directos, lo cual, además de estimular e intensificar el trabajo de la tierra, hace que la justicia vaya llegando a todos los hogares [... Sólo esto] ha de labrar la historia de nuestra independencia económica." 35 Esta recurrente retórica y exposición con eslóganes remite a las frases utilizadas por el peronismo: "la tierra para quien la trabaja" y "Mejor que decir es hacer y mejor que hacer es realizar".

Aquella proposición se basa en el fomento rural, con la elevación del standard de vida de esa población mediante el mutualismo y la cooperación ${ }^{36}$

características de cada una de las ciudades (Santa Rosa posee "bodegas importantes y es el centro comercial de una gran zona agrícola e industrial” y Los Berros "es el asiento de innumerables fabricantes de cal"). El diputado puntualiza que son "localidades entregadas al trabajo y a las transacciones comerciales" lo que hace indispensable una "comunicación rápida que las facilite". Este proyecto de ley pasa a la comisión de Comunicaciones y Transportes. Reunión $24^{\circ}$, Proyecto de ley XIX. Honorable Cámara de Diputados, 1946.

33Discurso presidencial ante Honorables Cámaras de Diputados y Senadores. Perón 26 de junio de 1946

34Discurso presidencial ante Honorables Cámaras de Diputados y Senadores. Perón 26 de junio de 1946

35Discurso presidencial ante Honorables Cámaras de Diputados y Senadores. Perón 1 de mayo de 1947

36 "Una amplia organización de cooperativas de productores en forma federada con el fin de proceder en colaboración con el Estado, asegurará la colocación en el mercado de los productos obtenidos". Discurso presidencial ante Honorables Cámaras de Diputados y Senadores. Perón 26 de junio de 1946 
Por su parte en relación a los servicios de comunicaciones, el enunciador hace una doble exposición: por un lado, recuerda que fueron adquiridos por el Estado recientemente y "[...] ha dejado de pertenecer a manos extranjeras y constituye una empresa mixta totalmente argentina al servicio de los argentinos"37, es decir, estos servicios fueron re-estatizados por obra de gobierno. Por otro lado, usa metáforas organicistas para dar idea de la importancia de la comunicación para país como unidad: esta empresa es una "parte integrante del completo organismo que representa la vida del país" 38 y son "el verdadero sistema nervioso del territorio argentino"39, esta unificación mediante la red de comunicaciones implica la representación de un tejido, en el que los sujetos aparecen enlazados entre sí.

Es terminante en sus afirmaciones "Los problemas que afectan a los territorios nacionales serán por fin encarados de manera efectiva por el gobierno nacional." ${ }^{40}$ En la misma línea, el primer mandatario destaca la obra de gobierno realizada en la Patagonia, ya que se crearon y pusieron en funcionamiento 12 establecimientos de educativos que "se ha hecho en esa zona en 11 meses más que en los anteriores 87 años" ${ }^{41}$. Aquí se puede hacer hincapié en otro punto central de los movimientos populistas ${ }^{42 .}$

Una temática característica del conjunto 1951-1955 aparece subrepticiamente en 1947. El líder argentino afirma que "en el concierto de las naciones, la Argentina no puede abastecerse a sí misma ni practicar ese aislacionismo suicida que fue la característica de otros tiempos y de otras naciones. Es necesario intensificar el intercambio comercial, cuidando de defender la producción nacional en el exterior y seguir una sana política en materia de importación de los productos esenciales para nuestra economía"43. Este posicionamiento se enlaza con la política y la acción del Instituto Argentino para la Promoción del Intercambio (I.A.P.I.) ${ }^{44}$.

37 Discurso presidencial ante Honorables Cámaras de Diputados y Senadores. Perón, 1 de mayo de 1947.

38 Discurso presidencial ante Honorables Cámaras de Diputados y Senadores. Perón, 1 de mayo de 1947.

39 Discurso presidencial ante Honorables Cámaras de Diputados y Senadores. Perón, 1 de mayo de 1947.

40 Discurso presidencial ante Honorables Cámaras de Diputados y Senadores. Perón, 1 de mayo de 1947.

41 Discurso presidencial ante Honorables Cámaras de Diputados y Senadores. Perón 1 de mayo de 1947.

42 “El gobierno anterior' hace de comodín en el mazo de las calamidades que asolan al desventurado pueblo", que el líder viene a redimir. Madriz, María Fernanda (2002), pag 84

43Discurso presidencial ante Honorables Cámaras de Diputados y Senadores. Perón 1 de mayo de 1947.

44 "Único comprador de nuestra producción agrícola y adquiriente en el exterior de las materias que son de vital importancia para nuestra economía, el instituto ajusta sus procedimientos comerciales a las cláusulas de los trabajos internacionales últimamente concertados. Coloca nuestra producción en el exterior y defiende los precios, asegura el intercambio para 
Siguiendo a Joseph Page, en las elecciones celebradas a comienzos de 1948 , el partido peronista obtuvo tan buenos resultados como en 1946. De esta manera, tenía la totalidad de las bancas del Senado y portaba una mayoría más amplia que la de los dos tercios en la Cámara de Diputados ${ }^{45}$.

Ahora bien, desde los discursos de apertura de Perón, se focaliza en otras cuestiones, por ejemplo, se invita a establecer fuentes de trabajo regionales para evitar la "despoblación del interior del país" ${ }^{46}$. Es importante reflexionar sobre el enorme arribo de personas, de familias enteras a las urbes (Gran Buenos Aires, por ejemplo) y consecuente problema habitacional, muy debatido en la Cámara de Diputados.

En 1949, se decide modificar la Constitución Nacional y pasa a llamarse Constitución Justicialista, aunque no todos los sectores estuvieron de acuer$\mathrm{do}^{47}$. Uno de los intelectuales encargados de su reforma, Arturo Enrique Sampay expuso en un libro de su autoría su predilección por la idea de bien común en el sentido tomista respecto a las principales teorías del Estado ${ }^{48}$.

Si bien el espíritu y la realización pueden dejar algunas zonas grises, la Nueva Constitución produjo varios cambios en la estructura política, permitió la re-elección del Presidente de la Nación, suprimió el sistema de electores, ahora las elecciones a presidente serían directas mediante el voto popular, así

los próximos cinco años y destina el margen de sus operaciones de compra-venta a la integración de un capital básico para sostener si llegara el caso, los precios internos de nuestra producción agropecuaria" (Discurso presidencial ante Honorables Cámaras de Diputados y Senadores. Perón 1 de mayo de 1947) El I. A. P. I. se encargaba de las exportaciones agropecuarias y de la compra de la producción a los sectores agrarios a precios establecidos por el Gobierno. Los productos eran vendidos en el mercado exterior a precios allí fijados. El resultado era un importante ingreso de divisas, cooptadas por el Estado para la expansión industrial, y del mercado interno, dado el mayor ingreso de los trabajadores.

45 Page, Joseph. (1984), pag 237. Además sostiene el autor que en "1948, el estado de ánimo en la Cámara de Diputados había cambiado perceptiblemente del casi inocente impromptu que caracterizó el primer período a un servilismo genuflexo respecto de la Casa Rosada. La discusión de los méritos de la legislación presentada se hizo menos frecuente y el tiempo dedicado a rendir homenajes a Perón y Evita aumentó. La asunción de Héctor Cámpora a la presidencia de la Cámara aceleró este proceso de deterioro" (Page, Joseph. (1984), pag 247) 46 Para Juan Domingo Perón: “El éxodo de grandes contingentes de población de la campaña hacia la ciudad, único lugar donde las posibilidades de la industria les ofrecían en tiempos pasados mejores condiciones de vida, se debió a la falta de arraigo del individuo a la tierra que fecundaba con su esfuerzo" (Discurso presidencial ante Honorables Cámaras de Diputados y Senadores. Perón 1 de mayo de 1948)

47 “La minoría radical del Congreso, frente a la desagradable opción de someterse o renunciar, eligió lo que le parecía el mal menor e hizo lo que se le exigía [juraron obediencia]. El presidente asistió a una sesión conjunta del Congreso el 15 de marzo y juró formalmente hacer cumplir la constitución reformada. Domingo Mercante fue quien le tomó el juramento." (Page, Joseph. (1984), pag 244) 48Sidicaro, Ricardo (2002), pag 96. 
como se modificó el Preámbulo49. En adelante la elección presidencial se haría en forma directa por el voto popular. Asimismo el número de ministerios se incrementaba de ocho a veinte. Es destacable la inclusión de derechos del trabajador y de la ancianidad, entre otros, dado que será legislación que entre en vigencia en todo lo ancho del país, unificándolo desde el marco legal.

Uno de los problemas que trajo la reforma fue la dificultad para el arribo del capital extranjero y su radicación en el país, dado que su "artículo 40 especificaba que el Estado podría intervenir legalmente en algunos sectores de la economía con el objeto de salvaguardar los intereses generales y dentro de los límites fijados por los derechos fundamentales que la Constitución le asignaba" (Di Tella y Zymelman, Las etapas del desarrollo económico argentino, Buenos Aires. EUDEBA, 1967). ${ }^{50}$ Motivo por el cual las empresas extranjeras no parecían convencidas de invertir en el país con esta salvedad constitucional, lo que impedía a su vez un desarrollo sostenido de la nación.

En el discurso de apertura de las sesiones ordinarias de 1949, Perón se detiene en comentar los estudios geológicos en determinadas zonas del país. Otro servicio del que se ocupa es de los transportes y comunicaciones pero sólo dice lo que se realizó en Gran Buenos Aires y Capital Federal. ${ }^{51}$

Tal como se había explicitado el año 1950 representa el inicio del eclipse peronista. Por un lado, porque el presidente de la Nación en la apertura de sesiones plantea la representación de un nuevo federalismo práctico en el marco de la "Nueva Argentina" y por otro, porque se corresponde con la advocación del año del Libertador General San Martín apelándose al pasado fundacional de la Nación. Además, presenta algunos indicios que se continúan en el segundo bloque de discursos -1951 a $1955^{-52 .}$

El nuevo federalismo práctico aporta propuestas claras para la acción y el cambio de mirada respecto del país: "Nuestro sistema federal ha sido por nosotros respetado, afianzado y consolidado. Siempre en procura de nuestra primera finalidad, la unidad nacional, hemos pensado que ella no sería posible sin

49El texto permaneció intacto, salvo el deseo de lograr una "Nación socialmente justa, económicamente libre y políticamente soberana”. Constitución de la Nación Argentina (2005), pag 77.

50Sidicaro, Ricardo (2002), pag 85.

51Discurso presidencial ante Honorables Cámaras de Diputados y Senadores. Perón 1 de mayo de 1949

52 "[... en el orden político, nuestro movimiento tiene la finalidad básica desde sus instantes iniciales: la unidad nacional”. Somos "una Nación políticamente soberana desde La Quiaca hasta la Antártida y desde los Andes hasta las Malvinas". Otra característica que se observa es la diferenciación respecto de procesos previos, con el consiguiente señalamiento de los dirigentes que estaban antes en el poder como "ellos", "los otros": "La verdad es que el federalismo que ellos proclamaron [... ] no fue sino un pretexto político para seguir explotando a las provincias como caudillos del mismo modo que explotaban a sus peones en las estancias" (Discurso presidencial ante Honorables Cámaras de Diputados y Senadores. Perón, 1 de mayo de 1950) 
poner previamente en pie de igualdad a todas las provincias frente al gobierno central. [...] El federalismo práctico que tiene sus bases en el ordenamiento económico de la Nación [...] tiene un fundamento económico que es técnicamente inobjetable. Cuando logremos su total realización se verá cómo no puede darse otro federalismo más efectivo dentro de nuestra unidad nacional" 53 . Vale destacar la utilización del nosotros, como parte de la lógica identitaria, por un lado y del discurso populista, por otro ${ }^{54}$.

La propuesta encara el logro de la unidad nacional para efectivizar la justicia social en todo el país. ${ }^{55}$ El orador puntualiza que "cada provincia argentina tiene en sí misma riquezas inagotables", a partir de esta posición explica que el "Plan Integral de Trabajos Públicos tiende a incorporar esas riquezas a la actividad del país", y argumenta, finalmente, que se realizaron acciones "[...] asegurando un 20\% de obras al Gran Buenos Aires y un $80 \%$ al interior del país." ${ }_{56}$

Noemí Girbal-Blacha observa que "el peronismo, a partir de 1950, revisa su política económica e incorpora un mayor número de prácticas liberales a ella [...] se acentúa progresivamente el crédito a favor del agro, a cuyos diferentes sectores nunca excluyó de sus beneficios" ${ }^{57}$.

Los discursos del presidente cambian el tono luego de 1950: aparece muy claramente el uso de "antes (del movimiento)" y "ahora (nosotros inclusivo; allí también ubica al proto-destinatario ${ }^{58}$ )", como demostración de la diferencia con otros gobiernos. El pueblo, los descamisados, los trabajadores están en la base de la idea de unidad nacional.

El giro realizado en la posición del enunciador, iniciado con algunas marcas en el discurso de 1950, se mantiene hasta 1955. El primer mandatario se posiciona en el discurso de inicio de las sesiones ordinarias en un "nosotros" y define los lineamientos tomados hasta la fecha (reformas económicas, políticas y sociales) ${ }^{59}$. Se puede observar que en esta segunda etapa, de 1951 a 1955, hace mayor referencia a la cuestión nacional.

Es relevante en esta segunda etapa el aporte social que se genera desde la Fundación María Eva Duarte de Perón de Asistencia Social (con personería jurídica desde 1948). La construcción de hospitales, policlínicos, escuelas, así como "la construcción de los barrios de viviendas de Corrientes, Santiago del

53Discurso presidencial ante Honorables Cámaras de Diputados y Senadores. Perón, 1 de mayo de 1950

54Martín Rojo, Luisa (1997) y Verón, E. - Sigal, S. (2003)

55Discurso presidencial ante Honorables Cámaras de Diputados y Senadores. Perón, 1 de mayo de 1950

56Discurso presidencial ante Honorables Cámaras de Diputados y Senadores. Perón 1 de mayo de 1950

57Girbal - Blacha, Noemí. (2002), pag. 11

58 Verón, Eliseo. (1985), pag. 17

59Discurso presidencial ante Honorables Cámaras de Diputados y Senadores. Perón, 1 de mayo de 1951 
Estero y San Luis, y en 1951 se terminarán las obras de Salta y de Catamarca"60, fomentan la idea de Nación como un todo. Hasta decir que "queremos también que ese esfuerzo llegue al agro argentino a fin de que los sacrificios y rigores del campo sean compensados por una vida sana y alegre en viviendas confortables y dignas" 61

Esta perspectiva demuestra una mirada de la "Nueva Argentina" como unidad. Asimismo distingue entre gobierno, Estado y pueblo pero en el marco del federalismo referido; sostiene que entrega "un gobierno centralizado que entiende el federalismo con sentido práctico [...] y que por eso gobierna a todo el país y a todos los argentinos" 62

Para dar cuenta de lo que se puntualizaba acerca de los gobiernos populistas, el presidente Perón frente a las Cámaras de Diputados y Senadores, en 1951 dijo: "El pueblo quiere que lo conduzcamos hacia la total realización de nuestra doctrina justicialista. Nosotros sólo cumplimos el mandato del pueblo."63

A partir de 1951, queda reflejado en el discurso la 'Vuelta al Campo'. El enunciador líder lo señala al decir que "nuestra producción agropecuaria [...] ha variado substancialmente sus antiguos horizontes", observa que se realizó "una intensa campaña de fomento de la producción agropecuaria, de cuyos resultados me considero profundamente satisfecho" "64, además se erige el yo como persona en el discurso ${ }^{65}$. El orador sostiene que "la curva de la producción agraria que venía descendiendo desde hace una década, ha retomado el camino ascendente y en la medida necesaria para satisfacer nuestro consumo interno progresivamente mayor, nuestra creciente industria y nuestro mercado internacional cada vez más diversificado, factores que exigen naturalmente una mayor producción." 66

Afirma que su gobierno desde 1950, "pusimos a disposición de nuestros campos todos los recursos de nuestra organización económica: nuestras mejores semillas; nuestros medios de lucha contra las plagas; el sistema bancario con sus ventajas crediticias; nuestras divisas para adquisición de maquinarias

60 Discurso presidencial ante Honorables Cámaras de Diputados y Senadores. Perón, 1 de mayo de 1951

61 Discurso presidencial ante Honorables Cámaras de Diputados y Senadores. Perón, 1 de mayo de 1951

62 Discurso presidencial ante Honorables Cámaras de Diputados y Senadores. Perón, 1 de mayo de 1951

63 Discurso presidencial ante Honorables Cámaras de Diputados y Senadores. Perón 1 de mayo de 1951. Cf. -Madriz, María Fernanda (2002)

64 Discurso presidencial ante Honorables Cámaras de Diputados y Senadores. Perón 1 de mayo de 1951.

65 El líder utilizaría el dirigirse en primera persona, “desde el Yo, individualiza el mando, singulariza el compromiso, personaliza en su única acción y decisión el acto de gobernar a los otros" (Madriz, María Fernanda. (2002), pag 77, 78, 79)

66Discurso presidencial ante Honorables Cámaras de Diputados y Senadores. Perón 1 de mayo de 1951. 
agrícolas; precios anticipados a la siembra misma; nuestro sistema de elevadores, nuestros transportes, nuestros silos, nuestros puertos, nuestros barcos, nuestros seguros y nuestro sistema de comercialización interna e internacional, etc." ${ }^{67}$.

Así cobra circulación y mayor sentido el slogan: "la tierra para el que la trabaja" ${ }^{68}$ es muy interesante que aquella expresión casi al pasar de 1946 acerca de organizar cooperativas regrese en $1951 .{ }^{69}$

Los discursos de apertura de sesiones legislativas presentan aquellas asignaturas pendientes que se deben realizar en el año de sesiones ordinarias que comienza. Pero también los objetivos que ya se lograron y sus delimitaciones, en este caso y en este momento, doctrinarias, son temas de agenda política y económica. Dicho esto, la cuestión de la descentralización fabril y el plan energético ${ }^{70}$ son dos tópicos claves del periodo, ya que posibilitarían la futura organización de "las zonas de la industria mediana y empiezan a alentarse programas extraordinarios que cimentarán la industria pesada nacional" "11. Son claramente una apuesta estrategia geopolítica.

En el discurso de 1951, el líder peronista cuenta que desde 1946, desde el gobierno de la Nación ha colaborado con las provincias "con una suma cercana a los mil millones de pesos, lo que da una idea de cómo entendemos nosotros al federalismo"72. Remarca que su gestión tiene ahora en sus manos la dirección bancaria y el control del comercio exterior, asegurando "a las provincias los recursos necesarios para materializar sus planes de obras, para ello ha organizado la financiación de las mismas a través del Consejo Federal Coordinador. Nosotros hemos cerrado, así todos los caminos por los que antaño se realizaba la entrega nacional."

Y finaliza subrayando que: "desde nosotros en adelante, solamente el gobierno nacional puede concertar empréstitos en el exterior. Y eso no lo hemos hecho todavía. ¡Ni lo haré yo en el gobierno! ¡Y no pienso dejarle tampoco ese compromiso a quien deba sucederme!"73. Es interesante la afirmación por-

67Discurso presidencial ante Honorables Cámaras de Diputados y Senadores. Perón 1 de mayo de 1951

68Discurso presidencial ante Honorables Cámaras de Diputados y Senadores. Perón 1 de mayo de 1951

69“Los productores agrarios deberían agruparse en cooperativas”. (Discurso presidencial ante Honorables Cámaras de Diputados y Senadores. Perón 1 de mayo de 1951)

70Yacimiento de petróleo, de gas, de carbón. “El suministro de gas desde Comodoro Rivadavia permitió la extensión de los servicios de gas envasado al interior del país" (Discurso presidencial ante Honorables Cámaras de Diputados y Senadores. Perón 1 de mayo de 1951) 71 Discurso presidencial ante Honorables Cámaras de Diputados y Senadores. Perón 1 de mayo de 1951

72Discurso presidencial ante Honorables Cámaras de Diputados y Senadores. Perón 1 de mayo de 1951

73Discurso presidencial ante Honorables Cámaras de Diputados y Senadores. Perón 1 de mayo de 1951 
que se había tomado un empréstito en $1950^{74}$ con el Eximbank de los Estados Unidos -préstamo por 125 millones de dólares destinados a saldar las deudas con los comerciantes norteamericanos- y el Presidente argentino será duramente criticado.

Perón destaca la "fecundidad del movimiento peronista" puesto de manifiesto con el gobierno federal y la consecución de "los grandes objetivos de nuestro afán: la grandeza de la Nación y la felicidad de los argentinos"75. Para demostrar este crecimiento ejemplifica a su audiencia con pares de opuestos: “[...] entre el peronismo y el anti-peronismo, entre la confabulación bradenista y la soberanía nacional, entre la prensa sirviente del capitalismo, y la verdad; entre la oligarquía y los descamisados, entre la patria y la traición"76.

La apertura de sesiones de 1952 presenta cambios como la participación activa de la mujer y de los representantes de los territorios nacionales, Chaco -Presidente Perón- y La Pampa -Eva Perón-77. Este discurso es marcadamente más doctrinario: plantea como una suerte de filosofía, una forma de vida, un fundamento del modo y las razones para hacer las $\operatorname{cosas}^{78}$. El mismo primer mandatario lo dice: "En este mensaje -destinado a fijar, sobre todo, los principios generales de nuestra doctrina-[...]"79.

El 1 de mayo de 1952, Perón, frente a las Cámaras sintetiza que "los beneficios del primer Plan Quinquenal han llegado a todos los rincones" 80 y destaca que las inversiones de este plan se dividieron el $75 \%$ al interior de la República y el $25 \%$ en el Gran Buenos Aires, pero no siempre las cifras absolutas lo respaldan. Sin embargo, en 1952 se implementó un Plan de Emergencia Económica para superar la sequía y la difícil conjetura internacional. ${ }^{81}$

74Girbal-Blacha, Noemí (1997)

75Discurso presidencial ante Honorables Cámaras de Diputados y Senadores. Perón 1 de mayo de 1951

76Discurso presidencial ante Honorables Cámaras de Diputados y Senadores. Perón 1 de mayo de 1951

77Entre ellos uno de los más evidentes es el saludo a "Señoras y señores", y se repite en 1953 y 1954, que revela la inclusión de la mujer argentina en la vida cívica: puede elegir y ser elegida. Asimismo la participación de delegados de los territorios nacionales que se incorporan por primera vez al Congreso de la República (Discurso presidencial ante Honorables Cámaras de Diputados y Senadores. Perón, 1 de mayo de 1952)

78“La causa del justicialismo, en sus aspectos económicos es la justa distribución de la riqueza entre los hombres"; "Para la doctrina justicialista todos los bienes económicos fueron creados y se crean y existen para el hombre"; "[nosotros] sostenemos que la economía y el Estado deben servir a la felicidad humana sirviendo al bienestar social" (Discurso presidencial ante Honorables Cámaras de Diputados y Senadores. Perón, 1 de mayo de 1952)

79Discurso presidencial ante Honorables Cámaras de Diputados y Senadores. Perón, 1 de mayo de 1952

80Discurso presidencial ante Honorables Cámaras de Diputados y Senadores. Perón, 1 de mayo de 1952

81Ricardo Sidicaro explica que "en la medida en que para asegurar la continuidad del proceso de industrialización en condiciones de deterioro de los precios agrarios internacionales eran 
Ya en 1953, "el Congreso Nacional y todas las legislaturas provinciales aprobaron el Segundo Plan Quinquenal de la República y en esas circunstancias se realizó el análisis exhaustivo de la situación económica, social y política del país"82. El gobierno peronista apuesta fuertemente a esta segunda planificación, para alcanzar "la autarquía del país" 83 , que empieza a conquistarla "con el apoyo técnico y financiero del Estado" ${ }^{84}$, así como con el financiamiento del Banco de la Nación y los beneficios como semillas seleccionadas, control de plagas, etc. Aunque Perón destaque que circula una propaganda antinacional y antiamericana, afirma que: "La revolución peronista, el movimiento peronista, la doctrina peronista, sólo llevan un nombre y un apellido que los identifican por su origen [...] pero todos sus principios y todas sus realizaciones ya se han convertido en principios y realizaciones de carácter nacional"85. Es interesante observar en el discurso la homologación entre Perón como enunciador y la construcción de la nación ${ }^{86}$.

La integración y la constante homogenización desde los primeros años del peronismo es planteada por el conductor Perón: "Las provincias han sido también beneficiadas en sus finanzas presupuestarias por esta reactivación agrícola ganadera, y en menos de dos años, con una más ordenada y eficiente organización de las recaudaciones [...] la mejor situación económica del sector agrario les ha permitido [así como el aporte del segundo plan quinquenal] ordenar sus presupuestos deficitarios, cancelar sus deudas e iniciar, aun antes que el propio gobierno nacional, y con ritmo acelerado de ejecución, las obras previstas en el segundo plan quinquenal. ¡Este es el sentido del auténtico federalismo que venimos practicando según nuestros principios fundamentales de

necesarios mayores saldos exportables, Perón solicitó en 1952, la colaboración de los productores rurales al anunciar las nuevas cotizaciones que pagaría el ente oficial [Plan Económico de 1952]. Los déficit del I. A. P. I. evidenciaron muy rápido los límites del proyecto peronista con respecto a la introducción de modificaciones en la estructura de la propiedad agraria y de su deficiente nivel de productividad." (Sidicaro, Ricardo (2002), pag 71)

82 Este plan ofrece medidas más liberales que el primero, una política agraria de subsidio y apoyo a los productores y de fomento a la producción agropecuaria mediante el Instituto Argentino de Promoción del Intercambio. Con este Plan se pretendía obtener mayores saldos exportables, para así alcanzar más divisas y pasar a la industrialización pesada. Y se esperaba la racionalización de la economía mediante una mayor eficacia, el aumento de la productividad laboral, proponiendo disminuir el consumo y aumentar el ahorro. Este segundo plan pasó a una relativa apertura de la economía, ya que se incentivaron las inversiones de capital extranjero en el desarrollo industrial y petrolero (dada la crisis), medidas que implicaron un abandono parcial del nacionalismo económico y del dirigismo estatal de la economía.

83 Discurso presidencial ante Honorables Cámaras de Diputados y Senadores. Perón, 1 de mayo de 1951

84 Discurso presidencial ante Honorables Cámaras de Diputados y Senadores. Perón, 1 de mayo de 1951

85 Discurso presidencial ante Honorables Cámaras de Diputados y Senadores. Perón 1 de mayo de 1953

86 Confrontar: Verón, E. - Sigal, S. (2003) 
unidad y solidaridad nacional!"87

Desde el Poder Ejecutivo, en 1954, se insiste, y se propone la ventaja de una industrialización regional específica para cada tipo de producción primaria. También se plantea la conveniencia del sistema de comercio interno de tipo cooperativo, especialmente si pertenecen a servicios sociales.

El Presidente de la Nación, en sus últimas alocuciones, regresa a la bandera de la tercera posición "porque tiene caracteres de solución universal, [... e] incluso, puede ser aplicada como solución humana a la mayor parte de los problemas del mundo" 88

En 1954, erige su punto de vista en "No somos ni seremos jamás políticos profesionales. La república está dividida: pero yo deseo la unión integral de todos los argentinos"89. Esta unión, tiene como fundamento la noción de integración y homogeneidad de la Nueva Argentina. Además focaliza en la separación de su construcción como sujeto en el discurso de los demás políticos, como sujetos mentirosos, casi despreciables ${ }^{90}$.

En relación al Plan Económico de 1952, Juan Domingo Perón considera que fue "oportuno y eficiente en sus determinaciones generales, y particularmente cuando estableció que el centro de gravedad de la acción económica a desarrollar estaba en el campo. El gobierno nacional y los organismos responsables del Estado, y los gobiernos provinciales en la órbita de su competencia, cumplieron el plan"91.

Es importante destacar que en la cosecha 53/54 se aplicó el denominado "sistema justicialista en el comercio de granos [como parte del sistema de economía social]. Y por primera vez también en la historia agraria de la República, los productores cobraron su cosecha en el acto de la entrega"92. En este plano, Perón vuelve a insistir sobre la ventaja de una industrialización regional específica para cada tipo de producción primaria, así como en la ventaja del sistema de comercio interno de tipo cooperativo y de las cooperativas en relación con las proveedurías. 93

Vinculado al afianzamiento de lo político y los actos cívicos, el llamado a

87Discurso presidencial ante Honorables Cámaras de Diputados y Senadores. Perón, 1 de mayo de 1954.

88Discurso presidencial ante Honorables Cámaras de Diputados y Senadores. Perón, 1 de mayo de 1954.

89Discurso presidencial ante Honorables Cámaras de Diputados y Senadores. Perón, 1 de mayo de 1954.

90 Verón, E. - Sigal, S. (2003)

91Discurso presidencial ante Honorables Cámaras de Diputados y Senadores. Perón, 1 de mayo de 1954.

92Discurso presidencial ante Honorables Cámaras de Diputados y Senadores. Perón, 1 de mayo de 1954.

93Discurso presidencial ante Honorables Cámaras de Diputados y Senadores. Perón, 1 de mayo de 1955. 
elecciones en el ex territorio de Misiones que "con ellas el pueblo de la nueva provincia ha constituido sus autoridades locales y cumplido la última etapa del proceso que iniciara con la sanción de la ley de provincialización por parte de vuestra honorabilidad [... para] brindarles los instrumentos necesarios para el pleno ejercicio de su autonomía"94

Ya para 1955, el orador espera la consolidación de las finanzas locales y se ha estructurado un "nuevo sistema de distribución de impuestos de coparticipación" 95 con el que la proporción que corresponde a las provincias aumenta del $32 \%$ a casi el $44 \%$ en el primer año de vigencia de la nueva ley. Perón explica que se lleva así al terreno de las realizaciones prácticas, su concepto del federalismo ${ }^{96}$.

\section{CONSIDERACIONES}

El movimiento peronista como un populismo con características diferenciales, genera un discurso político propio marcadamente más concreto que se abreva de lenguajes, códigos y discursos políticos previos ya reconocidos en el imaginario social, así, entra en interacción con un grupo social que aparecía marginado de la escena política hasta ese momento: los trabajadores. A su vez, el peronismo recreó otra lectura sobre qué era la República Argentina, ya que los desequilibrios regionales, por lo económico, lo social y lo político, generan tensiones en nuestro desarrollo como Nación. El peronismo aporta al debate un sistema de creencias, un cúmulo de ideologías que interaccionan con las ya existentes y se erigen.

Juan Domingo Perón tenía un poder otorgado por tanto por bases organizadas como por las masas. Llegó al gobierno contando con una cómoda mayoría en las Cámaras de Diputados y Senadores, de todos modos, el debate se podía desarrollar aunque no muchos legisladores peronistas supieran cómo era el funcionamiento correcto y normal de las Cámaras. Algunos autores citados remarcan que durante el primer mandato todos los días el presidente de la Cámara Baja se presentaba en Casa de Gobierno y le comentaba al Presidente de la Nación sobre las actividades en el recinto (leyes aprobadas, debates ocurridos), esperando también instrucciones.

La emergencia y la consolidación en el poder de este "nuevo" grupo, que tiene su mayor caudal de votos de las clases trabajadoras de todo el país, entre 1946 y 1951, enarbola el concepto de federalismo práctico con una consigna que envuelve a la Nación como unidad, como comunidad, en funcionamiento 94 Discurso presidencial ante Honorables Cámaras de Diputados y Senadores. Perón, 1 de mayo de 1955.

95 Discurso presidencial ante Honorables Cámaras de Diputados y Senadores. Perón, 1 de mayo de 1955.

96 Discurso presidencial ante Honorables Cámaras de Diputados y Senadores. Perón, 1 de mayo de 1955. 
con desarrollos regionales particulares.

Es importante destacar que Perón en sus discursos pasa de una concepción de nación como conjunto de regiones con cualidades particulares y riquezas infinitas, a la tensión: interior - Buenos Aires. Si bien hubo facilidades otorgadas a todas las regiones, la provincia de Buenos Aires, la Capital Federal y el Conurbano contaban con una infraestructura que desde el comienzo las posicionaba desde otro lugar y así se sostuvo desde el gobierno.

Hubo facilidades brindadas desde el Estado (semillas mejoradas, créditos, maquinaria, asesoramiento de especialistas) para afincar al agricultor y evitar el hacinamiento en los centros urbanos, sin embargo muchas familias para tener posibilidades de trabajar en una fábrica, por ejemplo, decidían instalarse en la región pampeana, o al menos en los centros urbanos.

En los bloques expuestos, con un quiebre marcado en 1950, Perón se mostró como un enunciador muy hábil, que sabía manejar su discurso y conocía el auditorio, que era diestro en el uso de estrategias comunicacionales y también de la diversidad de estilos para dirigir sus mensajes. Se puede afirmar, después de lo expuesto, que se está hablando de un populismo.

En la segunda parte de la gestión, el presidente Perón marca un cambio de rumbo que orienta el foco en la cuestión doctrinaria más que en la acción. El "nosotros" utilizado por Perón permite identificación con el enunciador, al tiempo que unifica y facilita la idea de "argentinos" como sinónimos de "peronistas". Así la aprobación de leyes y beneficios para los trabajadores, se observa un cambio no sólo desde el discurso sino también en la acción.

Respecto del Poder Legislativo, entre las leyes que fueron aprobadas se destacan las que facilitaban la construcción de escuelas, hospitales, viviendas, centros para niños y ancianos, puentes, usinas, correos, transportes, barrios, adquisición de teléfonos y trenes, bancos y la posibilidad de participación cívica de la mujer. En todos los casos se beneficiaron con el acceso generalizado a la educación, la salud, la vivienda y los créditos. Ciertamente, hubo restricciones en la libertad de acceso a la información y a la libertad de expresión y en el caso del Poder Judicial los jueces fueron desplazados de sus cargos, siendo las Cámaras las que juzgaron sus desempeños.

Los planes quinquenales posibilitaron la integración de la Nación mediante la realización de mejores comunicaciones, beneficios financieros y crediticios, así como la ayuda social desde el Estado. En consonancia, se nacionalizó parte de la economía -se "argentinizó", opina Perón en muchos discursos-, estatizándose los servicios públicos y los transportes, repatriando la deuda externa.

Es relevante el fomento de cooperativas "como unidades básicas justicialistas para la organización nacional de la producción, la industria y el comercio", porque estas instituciones son entidades sin fines de lucro y se organizan para la acción colectiva. Pareciera haber una búsqueda desde el Estado go- 
bernante para cooptar estas instituciones que, por sus lógicas constitutivas y modos de acción, poco tiene que ver con un aparato verticalizado, pero que les son funcionales a la hora de bajar los gastos de intermediación.

Las intervenciones federales resultan tema que se vincula con el espacio provincial y el "interior" y con sus normas. En estos casos extraordinarios el Estado Nacional (Poder Ejecutivo) decide, dados determinados conflictos institucionales o políticos, ir por sobre la autonomía provincial (inflingir el entramado federal). Así, en la década estudiada se intervinieron las provincias de Catamarca, Córdoba, La Rioja, Salta, Santa Fe y Santiago del Estero. Es importante destacar que en 1950, se realizó, por primera vez en el país una reunión plenaria de todos los gobiernos de provincias y de territorios. El peronismo desea que estas reuniones se desarrollen por "nuestro afán de servir lealmente, con claro sentido de unidad al federalismo, conciliándolo con los principios de la unidad nacional, nuestro supremo afán en el orden político"97

Del mismo modo es un dato central a remarcar la incorporación de los territorios: Chaco -Presidente Perón- y La Pampa -Eva Perón- a la participación con representantes en el Congreso ${ }^{98}$.

\section{REFERENCIAS}

ABOY CARLÉS, Gerardo (2005) Populismo y democracia en la argentina contemporánea. Entre el hegemonismo y la refundación, en Estudios Sociales $\mathrm{N}^{\circ} 28$, Santa Fe, Universidad Nacional del Litoral ÁLVAREZ JUNCO y GONZÁLEZ LEANDRI (1994) El populismo en España y América, Catriel. ARÉVALO CABEZA, Jabel. Proyecto de ley presentado por el diputado. Reunión $24^{\circ}$, Proyecto de ley XIX. Honorable Cámara de Diputados, 1946.

CONSTITUCIÓN DE LA NACIÓN ARGENTINA (2005). Producciones Mawis. 2da edición. DE IPOLA, Emilio (1980). Populismo e ideología: a propósito de E. Laclau. Teoría. Número 4. . (1983). Ideología y discurso populista. Buenos Aires. Folios.

DIARIOS DE SESIONES ORDINARIAS de la Cámara de Diputados de la Nación Argentina. 1946-1955.

DIARIOS DE SESIONES ORDINARIAS de la Cámara de Senadores de la Nación Argentina. 1946-1955.

GIRBAL-BLACHA, Noemí. (1997) "Cuestión regional - Cuestión nacional. Lo real y lo virtual en la historia económica argentina"; Notas y Comunicación; Ciclos en la Historia, la Economía y la Sociedad.

GIRBAL-BLACHA, Noemí. (1997) “Dichos y hechos del gobierno peronista (1946-55). Lo fáctico y lo simbólico en el análisis histórico", en Entrepasados. Revista de Historia. GIRBAL - BLACHA, Noemí. (2002) "Políticas públicas para el agro. Llamar al estado peronista (1943-1955)" Mundo Agrario, Revista de Estudios Rurales, número 5, 2do semestre, 97Discurso presidencial ante Honorables Cámaras de Diputados y Senadores. Perón, 1 de mayo de 1951. 98Discurso presidencial ante Honorables Cámaras de Diputados y Senadores. Perón, 1 de mayo de 1952. 
Centro de Estudios Históricos Rurales, Universidad Nacional de La Plata.

GIRBAL-BLACHA, Noemí. (2004) "Espacio regional, sujetos sociales, políticas públicas. Reflexiones históricas sobre el estudio de las continuidades y cambios de la Argentina rural". Estudios. Revista del Centro de Estudios Avanzados de la Universidad Nacional de Córdoba. JAMES, Daniel (2006) "Resistencia e integración. El peronismo y la clase trabajadora argentina. 1946-1976". Editorial Sudamericana. Primera parte. Los antecedentes. El peronismo y la clase trabajadora. $1943-55$.

LATTUADA, Mario (2002) El peronismo y los sectores sociales agrarios. La resignificación del discurso como articulador de los cambios en las relaciones de dominación y la permanencia de las relaciones de producción."El populismo como fenómeno multifacético" en Mundo Agrario. Revista de estudios rurales, $\mathrm{n}^{\circ} 5$, segundo semestre. Centro de Estudios Histórico Rurales. Universidad Nacional de La Plata, http://mundoagrarioold.fahce.unlp.edu.ar/nro5/ Lattuada.html MACKINNON, Moira. (2002) “Capitulo I. Introducción”. Los años formativos del partido peronista. Siglo XXI de Argentina Editores. Instituto Di Tella.

MADRIZ, María Fernanda (2002) La noción de pueblo en el discurso populista, Revista ALED MARTÍN ROJO, Luisa (1997) El orden social de los discursos, Discursos 21/22 PAGE, Joseph. (1984) Perón. Primera parte (1895-1952). Circulo de lectores. PARDO ABRIL, Neyra (2003) Análisis critico del discurso y representaciones sociales: un acercamiento a la compresión de la cultura. En Berardi, Leda (comp.) Análisis crítico del discurso. Perspectivas latinoamericanas. Santiago de Chile: Frasis editores. PERÓN, Juan D. 18/enero/1945. Límites del Estado. Pág. 34 RAITER, Alejandro (1994); La especificidad del discurso político en Elizaineín y Madfes (comp.), Análisis del discurso, Montevideo. Universidad de La República. RAITER, Alejandro (2002) Representaciones Sociales. Capítulo I, EUDEBA SIDICARO, Ricardo (2002) “Los tres peronismos. Estado y poder económico. 1946-55/197376/1988-99". 2. El Estado peronista y el cuestionamiento de los predominios de los principales actores socioeconómicos 1946-1955. Siglo XXI Editores.

VAN DIJK, Teun A. (2004) Ideología y discurso. Una introducción multidisciplinaria. . (2005) “Ideología y análisis del discurso" Revista Internacional de Filosofía Iberoa-

mericana y Teoría Social. Afil 10. № CESA - FCES- Universidad de Zulia Maracaibo - Venezuela. Abril/junio.

VERÓN, Eliseo. (1985) “La palabra adversativa. Observaciones sobre la enunciación política”. El discurso político, Buenos Aires, Hachette.

VERÓN, Eliseo - SIGAL, Silvia (2003) Perón o muerte. Los fundamentos discursivos del fenómeno peronista, EUDEBA. 


\section{Ximena A. Carreras Doallo}

Doutora em Ciências Sociais pela Universidad Nacional de Quilmes - UNQ, Mestre em Ciências Sociais e Humanas (UNQ), Especialista em Ciências Sociais e Humanas (UNQ) e Graduada em Comunicação Social (UNQ). Pertence ao Centro de Estudos da Argentina Rural - CEAR da UNQ. Professora no curso de Comunicação Social em UNQ. 